\title{
Does the interaction between vehicle headlamps and roadway lighting affect visibility? A study of pedestrian and object contrast
}

\author{
Rajaram Bhagavathula, Ronald Gibbons, Maury Nussbaum \\ Virginia Tech Transportation Institute
}

\begin{abstract}
Vehicle headlamps and roadway lighting are the major sources of illumination at night. These sources affect contrast - defined as the luminance difference of an object from its background - which drives visibility at night. However, the combined effect of vehicle headlamps and intersection lighting on object contrast has not been reported previously. In this study, the interactive effects of vehicle headlamps and overhead lighting on object contrast were explored based on earlier work that examined drivers' visibility under three intersection lighting designs (illuminated approach, illuminated box, and illuminated approach + box). The goals of this study were to: 1) quantify object luminance and contrast as a function of a vehicle's headlamps and its distance to an intersection using the three lighting designs; and, 2) to assess whether contrast influences visual performance and perceived visibility in a highly dynamic intersection environment. Both luminance and contrast of roadway visibility targets and a pedestrian were measured with a calibrated photometer at a realistic intersection. Both target and pedestrian contrast and luminance were substantially affected by the intersection lighting configuration, illuminance level, location at the intersection, and vehicle distance from the intersection. Objects also underwent changes in contrast polarity (positive to negative or vice-versa) as the distance between the vehicle and object changed. During these polarity transitions, objects became invisible because the contrast was zero. Negative contrast on targets was associated with higher visual performance. Within a given contrast polarity (positive vs. negative), visual performance depended on the magnitude of contrast, with higher contrast associated with higher visual performance. The relationship between pedestrian contrast and perceived visibility was complex, since pedestrians were often rendered in multiple contrasts. These findings have important implications for the lighting design of intersections and the development of nighttime pedestrian detection systems that rely on computer vision
\end{abstract}

\section{Introduction}

Vehicle headlamps and roadway lighting are the major sources of illumination at night. Both of these sources of illumination affect pedestrians' contrast at night. There are two kinds of contrasts relevant to visibility. The first is luminance contrast, which is the ratio of the luminance difference between an object and its background vs. the luminance of its background. The second is color contrast, which is the ratio of the color difference between an object and its background vs. the color of its background. In nighttime driving scenarios, the human visual system is under mesopic vision $[1,2]$ and drivers' color recognition capabilities (cones) do not play an important role. However, in this mesopic state of adaptation, rods Page 1 of 10 play an important role and luminance contrast drives an object's visibility more so than color contrast [3,4]. Therefore luminance contrast measures are most relevant and typically used in nighttime visibility research [5-9]. Luminance contrast, or Weber contrast, is defined as the ratio of the luminance difference between an object and its background relative to the luminance of the background, as shown in Equation 1:

$$
C=\frac{\left(L_{t}-L_{b}\right)}{L_{b}}
$$

where $C=$ luminance (Weber) contrast, $L_{t}=$ target luminance, and $L_{b}$ $=$ background luminance.

An object is considered be in negative contrast when it is darker than its background and positive contrast when it is brighter than its background. Contrast polarity is an interesting phenomenon that was detailed in early work by Aulhorn [10], as cited by Adrian [11], who reported that that objects in negative contrast are detected sooner than those in positive contrast for the same difference in luminance. This phenomenon was also reported by Hills [12], specifically under road lighting conditions; objects in negative contrast were detected from farther away than those in positive contrast. Object size also influences perceived visibility, with larger objects being perceived as being more visible than smaller objects [13]. In the presence of roadway lighting, object contrast also depends on several additional factors, which include target reflectance, vehicle headlamp type, location of the object with respect to the vehicle, and distance between the vehicle and the object $[6,14]$. However, the combined effect of intersection lighting design and vehicle headlamps on object contrast has not been previously reported to our knowledge, nor have changes in object contrast as a vehicle approaches an intersection.

To address these gaps, two studies were conducted that evaluated the effect of intersection lighting design on visual performance [15] along with perceived visibility and glare [16]. Intersection lighting design here refers to both the part of the intersection illuminated (lighting configuration) and the recommended illuminance level. Visual performance was measured using detection distance - the distance at which drivers detected a target located at the intersection. Perceived visibility and glare were measured using composite subjective ratings of pedestrian visibility, target visibility, intersection visibility, and glare. In the visual performance experiment [15], the lighting configuration that illuminated the intersection box (Box) yielded better visual performance (longer detection distances) and fewer missed target detections than the two alternatives tested (Approach and Approach + Box). Visual performance typically plateaued (no increase in visual performance with a corresponding increase in illuminance) between 8 and 12 lux 
illuminance levels. While there was no difference between the three lighting configurations in terms of pedestrian visibility, the Box lighting configuration resulted in higher perceived target and intersection visibility and lower perceived glare [16]. Perceived visibility ratings also plateaued between 8 and 12 lux illuminance levels in this lighting configuration.

Although these two experiments helped identify effective intersection lighting designs, they did not reveal why one design (i.e., Box configuration) resulted in better visual performance and higher perceived visibility than the rest. Changes in visual performance and perceived visibility between designs could be attributed to the contrast in which the objects (targets and pedestrians) were rendered by the intersection lighting designs. It is important to know how different intersection lighting design affect object contrast, since contrast drives the visibility of an object and object contrast is often directly proportional to its visibility $[6,11,17,18]$.

This study thus had two goals. The first goal was to quantify object luminance and contrast as a function of a vehicle's headlamps and its distance to an intersection using the three lighting designs. This quantification was done for three intersection lighting designs, as evaluated in the visual performance and the perceived visibility experiments. The second goal was to assess whether contrast influences visual performance and perceived visibility in a highly dynamic intersection environment. An object's luminance and contrast might change as vehicle approaches an intersection and the influence of headlamps increases [6, 14]. The location of an object with respect to the location of the luminaire at the intersection was expected to affect target luminance and contrast. Object contrast and size were also expected to influence visual performance and perceived visibility, similar to earlier evidence $[10,12,13]$. The results of this study were intended to supplement earlier results regarding visual performance and perceived visibility and glare, and to help identify underlying reasons for the superior performance of the Box intersection lighting configuration.

\section{Methods}

Photometric measurements were conducted at the intersection on the Virginia Smart Road at the Virginia Tech Transportation Institute. Photometric measurements included the luminance of objects and their backgrounds. Targets and a simulated pedestrian were used as objects in this study. The targets were gray in color, and $18 \mathrm{~cm}$ by 18 $\mathrm{cm}$ in size, similar to the ones used in the visual performance experiment [15]. Targets were located at entrances, exits, and the middle of the crosswalks at the intersection. Targets located at the entrances and exits were placed at distance of 0.3 meters outside the right shoulder. There were five target locations as described in [15] (Figure 1). The simulated pedestrian was same as the pedestrian described in the perceived visibility experiment [16] (Figure 2) and was located at the Near Right location (see Figure 1). Pedestrian location for photometric measurements was moved slightly to the left of the actual location used in the subjective ratings experiment, in interest of saving time and to facilitate capturing of all object locations with a single image. Since this move was lateral, the change in the light levels (luminance and illuminance) was minimal.

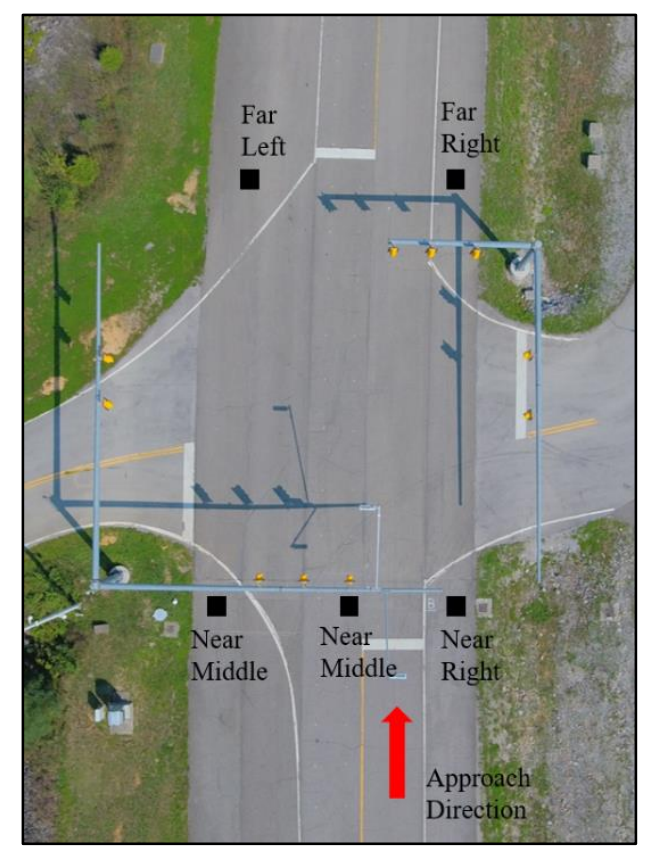

Figure 1. Target locations at the intersection

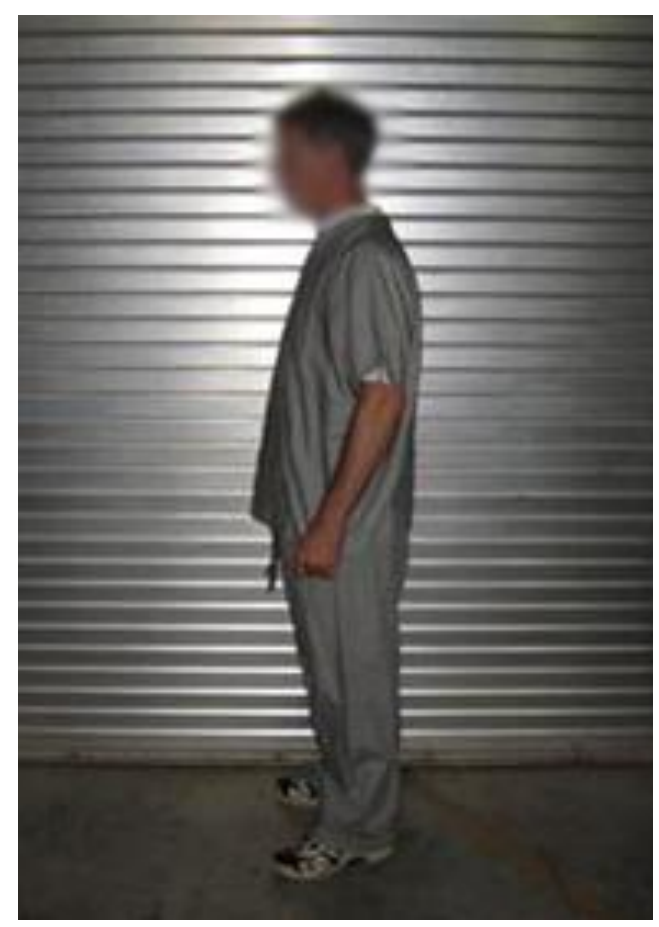

Figure 2. Pedestrian with grey scrubs

Lighting configurations and illuminance levels were similar to the ones used in the visual performance [15] and perceived visibility experiments [16]. Three lighting configurations were used. In the first lighting configuration, the approach to the intersection was illuminated (Approach, see Figure 3a). In the second, the intersection box was illuminated (Box, see Figure 3b), while in the third, both the approach and the box of the intersection were illuminated (Both, see Figure 3c). The intersection was illuminated by two 4000 Kelvin light emitting diode (LED) luminaires. One was used for illuminating the approach and the other was for illuminating the Box. The 
Approach luminaire had a type II distribution and the Box luminaire had a type $\mathrm{V}$ distribution. These luminaires had a mounting height of 8.5 meters ( $28 \mathrm{ft}$.). Four illuminance levels were used for the photometric measurements, specifically, 8, 12, 16 and 21 lux. These levels were the horizontal illuminance levels measured at the near right target location and were selected based on the IESNA RP-8 minimum recommended levels for low, medium and high pedestrian volumes at rural, sub-urban, and urban locations [19].

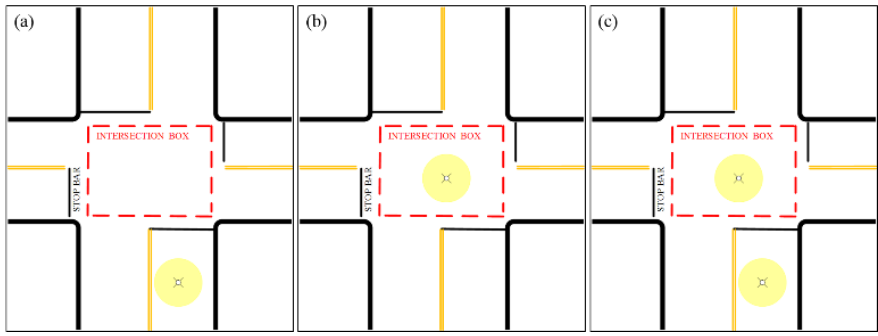

Figure 3. Illustrations of the three intersection lighting designs: (a) Intersection approach is illuminated; (b) Intersection box is illuminated; and (c) Both the box and approach are illuminated

Luminance and contrast of targets and the pedestrian were calculated from photometric images taken at 10 distances to the intersection, and in each of the three lighting configurations and four illuminance levels. These distances ranged from 120 to 20 meters to the intersection, at 10 meter intervals. The range of distances was selected to capture the luminance and contrast of the objects at the intersection as a vehicle approaches and the headlamps come into influence. Headlamps have an effective range that varies between 80 to 120 meters depending on the manufacturer [20]. From a prior study [21], it was evident that the range of the headlamps used here study was between 80 and 90 meters. The selected ranges thus helped in capturing object luminance and contrast before and after the influence of headlamps.

Photometric images were captured with a calibrated photometer (ProMetric PM-9913E-1, Radiant Imaging ${ }^{\circledR}$, Redmond, WA) mounted inside the test vehicle at the driver seat (Figure 4). A 2000 Ford Explorer was used as the test vehicle, one of the two experimental vehicles used in both the visual performance and the perceived visibility experiments. The vehicles' headlamps were Hella $90 \mathrm{~mm} \mathrm{Bi}-X e n o n$ projector lamps, with a single 1-F capacitorstabilized headlamp input voltage on each vehicle. These headlamps were retrofitted along with a voltage stabilizer so that the headlamps' intensities were not affected by vehicle speed. Captured images were analyzed using Radiant Imaging ${ }^{\circledR}$ ProMetric software (ver. 9.1,

Radiant Imaging ${ }^{\circledR}$, Redmond, WA). For calculating the luminance of targets and pedestrians, polygons were traced around the pedestrian and targets, and the software calculated the mean luminance within the selected polygon. Background luminance was calculated by tracing the same-sized polygon around the object location close to its boundaries. The Weber contrast formula (1) was used to calculate the contrast of the pedestrian and targets.

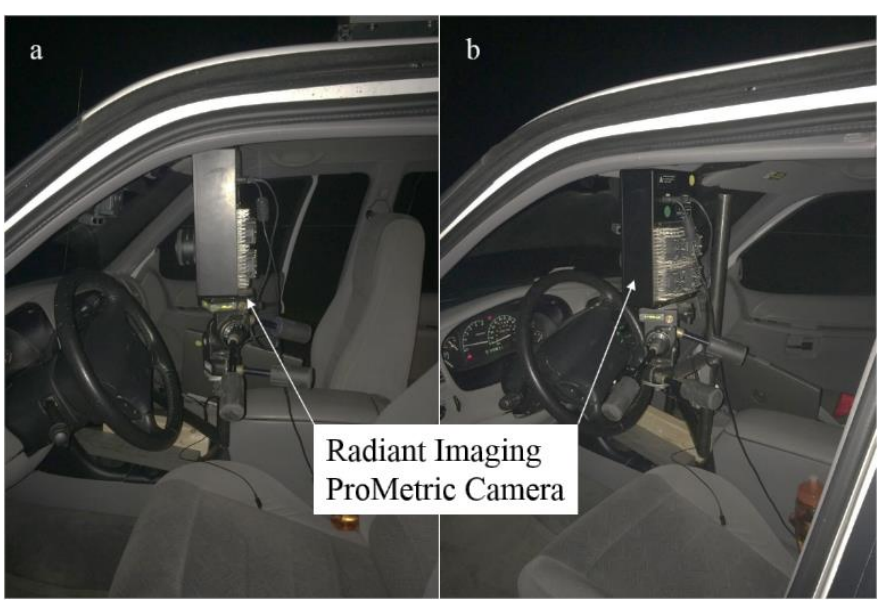

Figure 4. Location of the photometer inside the test vehicle at the driver's seat

Detection distances from the visual performance experiment were used to understand the relationship between target contrast and distance to the intersection. To simplify and facilitate the ease of understanding, target contrast at the mean detection distance under each lighting configuration and illuminance level were also graphed on contrast-distance to intersection plots. Contrast and distance to target data were interpolated to obtain the contrasts at the mean detection distances. For pedestrians, the contrast was calculated at 76.2 meters from the intersections and was graphed on the contrastdistance to intersection plots, which is the distance from where the participants rated pedestrian visibility in the perceived visibility experiment. The change in luminance and object contrast (targets and pedestrians) as the vehicle approached the intersection, along with the contrast at the mean detection distance and pedestrian visibility rating location, will help understand the relationship between object contrast, visual performance, and perceived visibility at the three intersections lighting configurations.

Two photometric evaluations were conducted. The first focused on the change in target luminance and contrast from the point of view of an approaching vehicle. This was done for each of the five target locations used in the visual performance experiment. Results of these evaluations were intended to help in understanding the relationship between target contrast, visual performance, and perceived visibility. These results could also explain why one intersection lighting configuration performed better than the other. Also in these evaluations, contrasts of the far-right and far-left target locations were compared at each illuminance level under the Box lighting configuration, to understand why the latter had longer detection distances than the former. The second evaluation focused on changes in pedestrian luminance and contrast from the point of view of an approaching vehicle. These evaluations helped understand the relationship between pedestrian contrast and their perceived visibility.

\section{Results}

\section{Target Luminance}

Increases in illuminance levels resulted in an increase in target luminance in all three lighting configurations (see Figure 5, Figure 6, Figure 7, Figure 8 and Figure 9). However, the rate of increase in the target luminance with distance depended on target location and lighting configuration. For the near right, near middle, and near left

Page 3 of 10 
target locations, the Approach lighting configuration had the highest target luminance at every distance to the intersection, whereas the Box lighting configuration had the lowest (Figure 5, Figure 6 and Figure 7). This trend reversed for the far right and far left target locations (Figure 8 and Figure 9).

In general, target luminance increased as the vehicle approached the intersection. Between 120 and 80 meters to the intersection, there were no major changes in target luminances at any of the target locations. However, for targets on the right shoulders (near right, far right) and the middle (near middle), at distances less than 80 meters to the intersection, there was a rapid increase in the target luminance with decrease in distance to the intersection (Figure 5, Figure 6 and Figure 8). This increase in target luminance was also observed for targets on the left shoulder (near left and far left), but the increase was modest (Figure 7 and Figure 9).
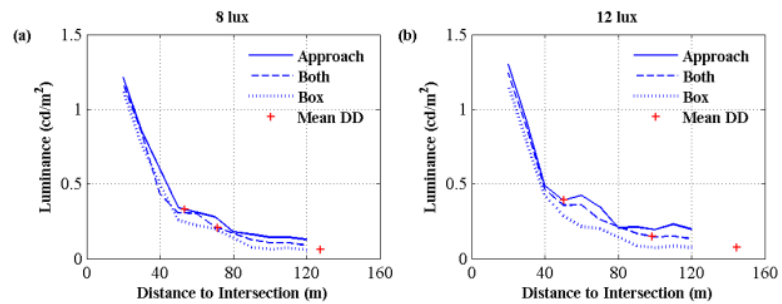

$16 \operatorname{lux}$
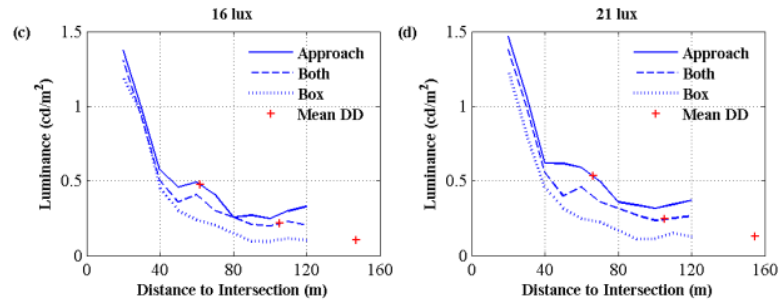

Figure 5. Luminance measurements at the near right target location as a function of the vehicle distance to the intersection in each lighting configuration and illuminance level. The "+" represents the luminance at the mean detection distance.
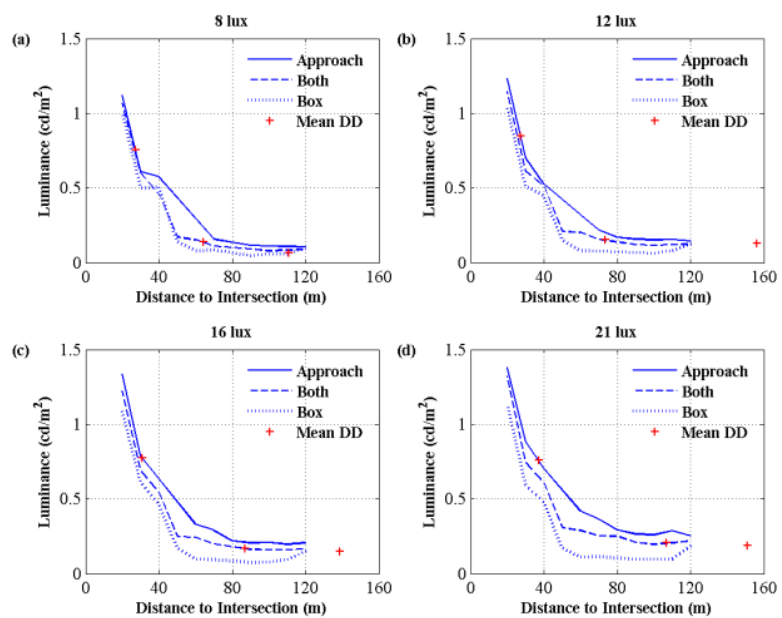

Figure 6. Luminance measurements at the near middle target location as a function of the vehicle distance to the intersection in each lighting configuration and illuminance level. The "+" represents the luminance at the mean detection distance.

Page 4 of 10
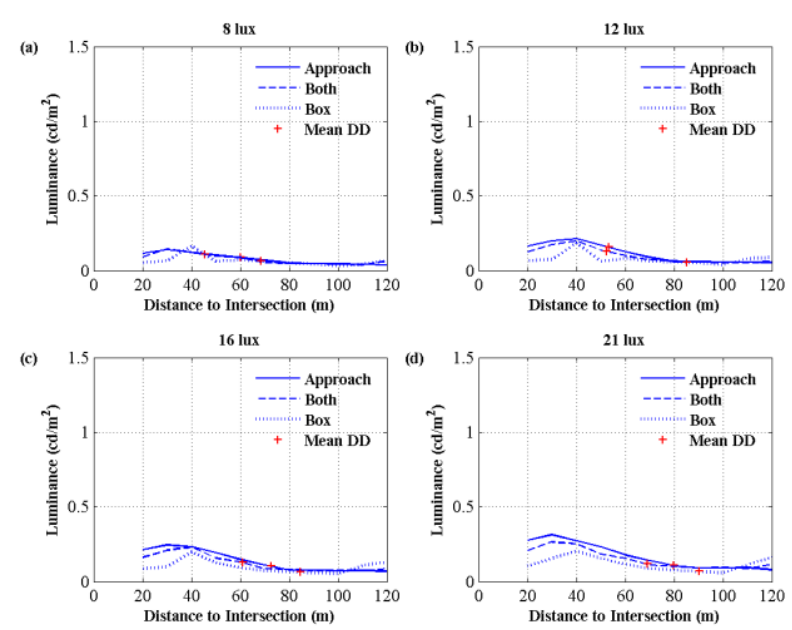

Figure 7. Luminance measurements at the near left target location as a function of the vehicle distance to the intersection in each lighting configuration and illuminance level. The "+" represents the luminance at the mean detection distance.
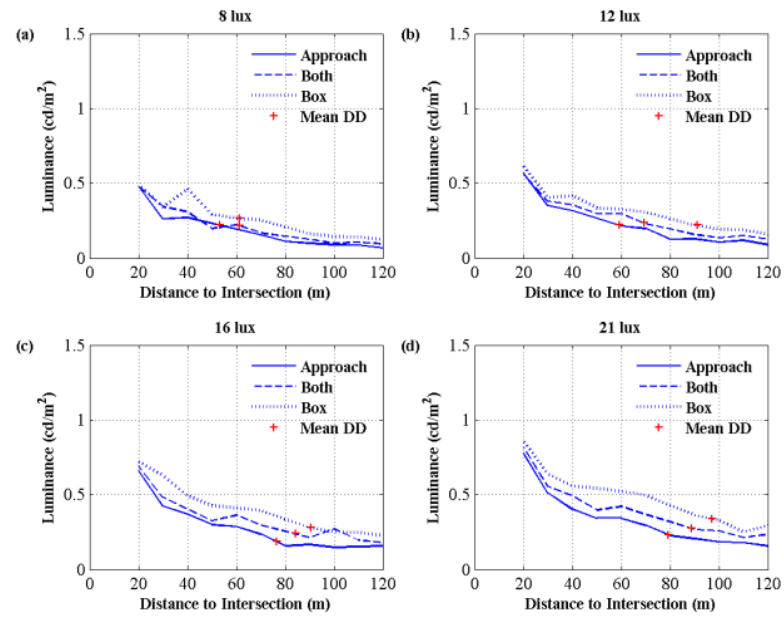

Figure 8. Luminance measurements at the far right target location as a function of the vehicle distance to the intersection in each lighting configuration and illuminance level. The "+" represents the luminance at the mean detection distance. 

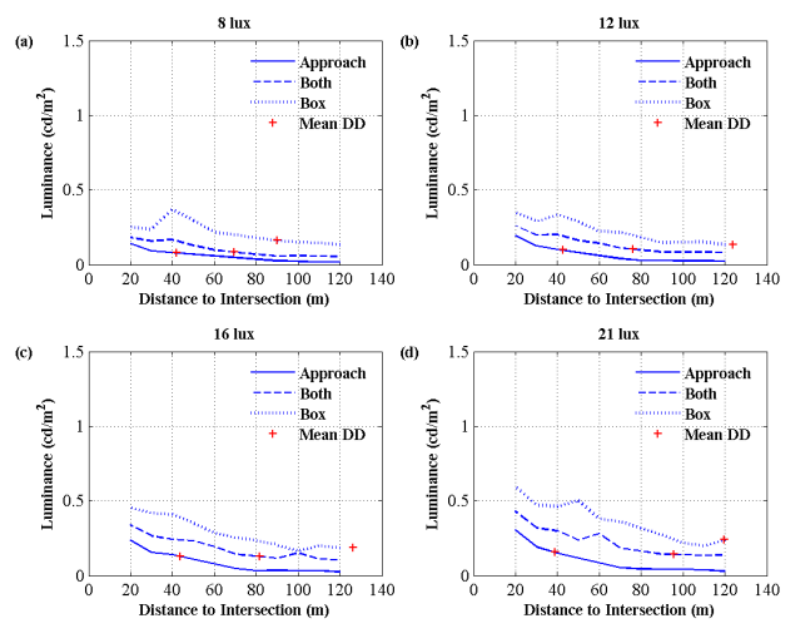

Figure 9. Luminance measurements at the far left target location as a function of the vehicle distance to the intersection in each lighting configuration and illuminance level. The "+" represents the luminance at the mean detection distance.

\section{Target Contrast}

For the Box lighting configuration, detections happened at distances longer than where the contrast measurements took place.

Consequently, detections are marked outside the range at the same level as the last measured contrast without any extrapolation. Target contrast was influenced by lighting configuration, illuminance level, and target location.

In the Approach lighting configuration, all the target locations were rendered in positive contrast, except the near right target at illuminance levels greater than 8 lux. In the latter, target contrast became negative before increasing to positive at distances shorter than 40 meters to the intersection. Target contrast decreased as the vehicle approached the intersection until about 40 meters to the intersection, after which it increased. Increases in target contrast with an increase in the illuminance level was only observed for the near left and far right target locations.

In the Box lighting configuration, the contrast polarity of the targets depended on their location and the distance from the intersection. Near right and near middle target locations were rendered in negative contrast, and as the vehicle approached the intersection the target contrast changed into positive contrast. The distance at which these targets changed into positive contrast depended on the illuminance level (Figure 10 and Figure 11). The remaining targets were rendered in positive contrast, and as the vehicle approached the intersection the magnitude of the contrast reduced (Figure 12, Figure 13 and Figure 14). In the case of the near left target, the target contrast transitioned from positive to negative contrast between 50 and 60 meters to the intersection (Figure 12). Increases in illuminance level was also associated with increases in the magnitude of the target contrast.

In the Both lighting configuration, the contrast polarity of the targets also depended on their location and the distance from the intersection. Near right and near middle targets started in positive contrast, which decreased with a decrease in distance to the intersection, until switching to negative contrast where it plateaued. As the vehicle got closer to the intersection, the contrast polarity again changed to positive. The magnitude and the distance at which Page 5 of 10 the target was rendered in negative contrast depended on the illuminance level (Figure 10 and Figure 11). The rest of the targets were rendered in positive contrast, and their contrasts decreased as the vehicle got closer to the intersection. For the near left target, the target contrast transitioned from positive to negative contrast between 20 and 60 meters to the intersection, depending on the illuminance level (Figure 12). This transition happened at longer distance at higher illuminance levels. Effects of increasing illuminance levels on the magnitudes of contrast depended on the target location. An increase in contrast with an increase in illuminance level was only observed for near left, far right, and far left locations (Figure 12, Figure 13 and Figure 14).

Targets rendered in negative contrast had longer mean detection distances than those rendered in positive contrast (Figure 10 and Figure 11). Within each contrast polarity, mean detection distance depended on the magnitude of the contrast. Higher contrasts were associated with longer detection distances (Figure 13 and Figure 14). In the perceived visibility experiment, perceptions of target visibility were assessed as a whole, and results regarding perceptions of individual target visibility were not available for direct comparisons with target contrasts. However, perceptions of target visibility overall were highest in the Box lighting configuration and lowest in the Approach lighting configuration. These perceptions are consistent with the relative levels of target contrasts reported here, with more negative contrast (for near right and near middle targets) and higher levels of positive contrast (for far right and far left targets) associated with higher perceived visibility.
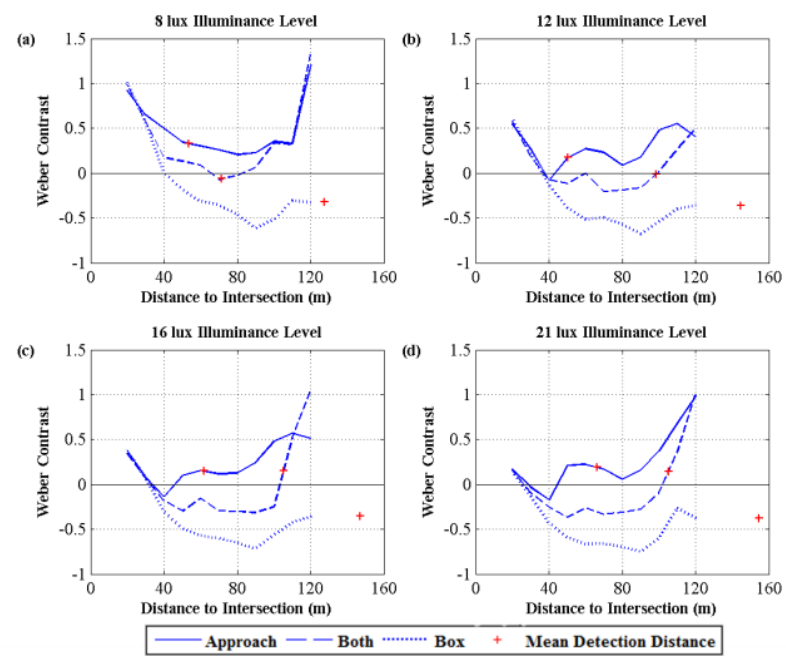

Figure 10. Target contrast at the near right target location as a function of the vehicle distance to the intersection in each lighting configuration and illuminance level. The "+" represents the contrast at the mean detection distance. 
(a)
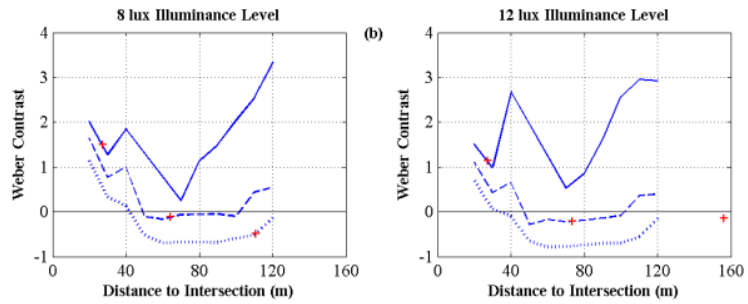

(c)

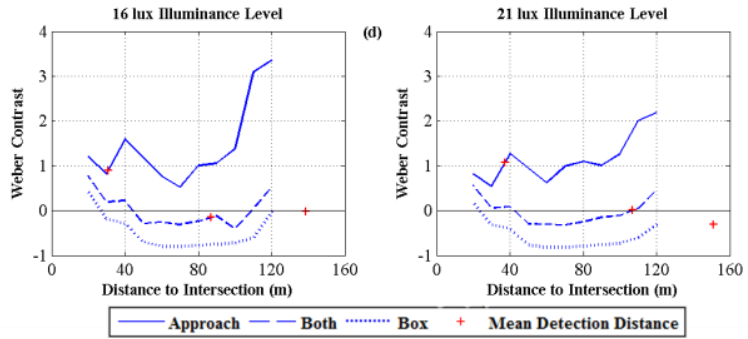

Figure 11. Target contrast at the near right middle target location as a function of the vehicle distance to the intersection in each lighting configuration and illuminance level. The "+" represents the contrast at the mean detection distance.

(a)
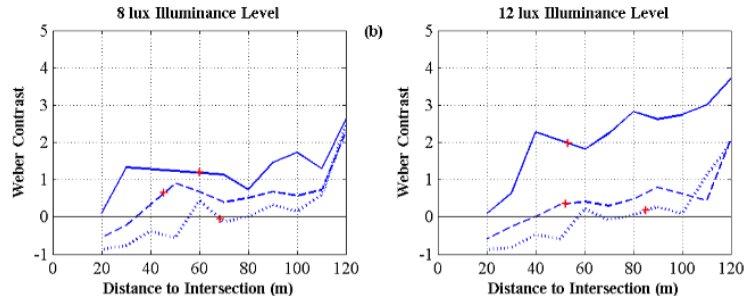

(c)
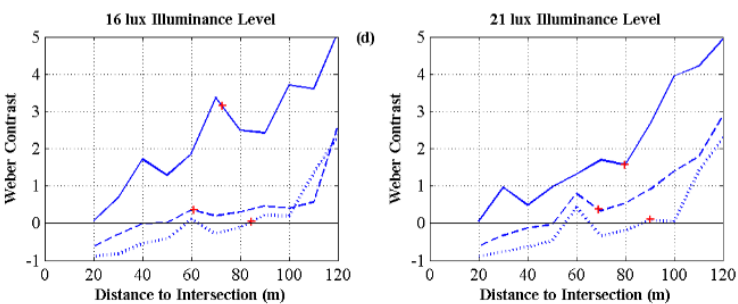

oth …….... Box

Intersection $(m)$

Figure 12. Target contrast at the near left target location as a function of the vehicle distance to the intersection in each lighting configuration and illuminance level. The "+" represents the contrast at the mean detection distance.
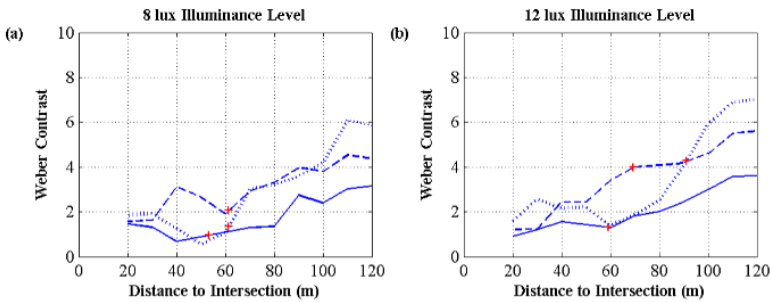

(c)

16 lux Illuminance Level
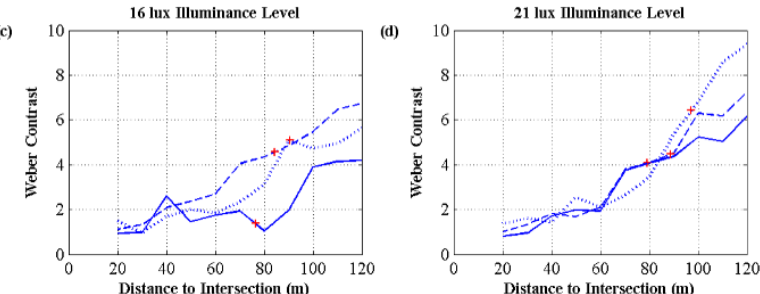

Approach -- Both ……… Box + Mean Detection Distance

Figure 13. Target contrast at the far right target location as a function of the vehicle distance to the intersection in each lighting configuration and illuminance level. The "+" represents the contrast at the mean detection distance. (a)

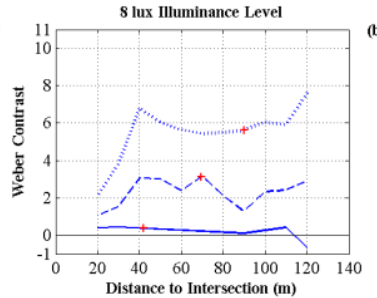

16 Iux Illuminance Level

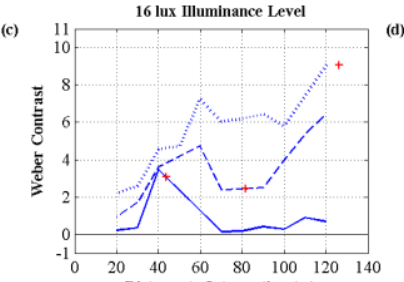

Distance to Intersection $(\mathrm{m})$

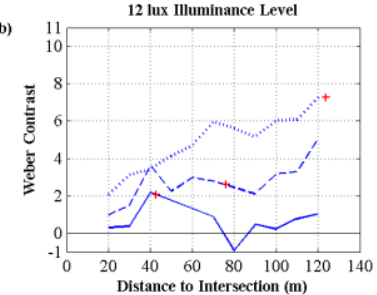

21 lux Illuminance Level

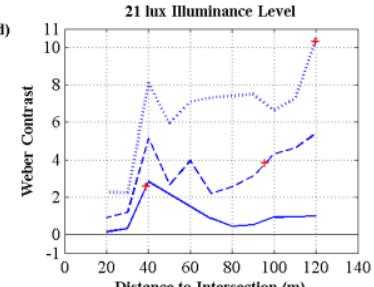

Distance to Intersection (m)
Figure 14. Target contrast at the far left target location as a function of the vehicle distance to the intersection in each lighting configuration and illuminance level. The "+" represents the contrast at the mean detection distance.

\section{Pedestrian Luminance}

The luminance of the pedestrian increased as the vehicle approached in all the three lighting configurations (Figure 15). An increase in illuminance level was also associated with an increase in the pedestrian luminance. Further, at every distance and illuminance level, pedestrian luminance was highest in the Approach lighting configuration and lowest with Box lighting (Figure 15). 

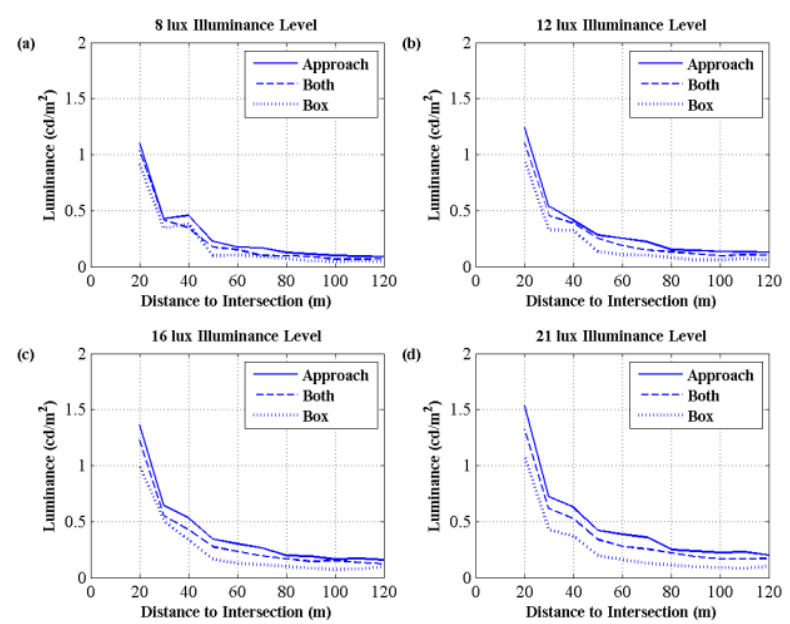

Figure 15. Pedestrian luminance as a function of the vehicle distance to the intersection.

\section{Pedestrian Contrast}

Pedestrian contrast varied with the distance to the intersection, lighting configuration, and illuminance level (Figure 16). In the Approach lighting configuration, the pedestrian was always rendered in positive contrast and the contrast decreased as vehicle approached the intersection, until about 40 meters. After 40 meters, the contrast sharply decreased at 30 meters to the object and then increases rapidly at 20 meters again. This behavior was consistent across all illuminance levels. In the Both lighting configuration, pedestrian contrast started positive and decreased until 80 meters. From 80 to 50 meters, contrast polarity depended on the illuminance level. At the lower illuminance levels ( 8 and 12 lux), it went into negative contrast and plateaued. At the higher illuminance levels (16 and 21 lux), the pedestrian was rendered in positive contrast and plateaued. At 40 meters, the contrast increased rapidly at all the illuminance levels and then decreased at 30 meters and increased again at 20 meters, similar to the contrast behavior in the Approach lighting configuration. In the Box lighting configuration, the pedestrian was rendered in negative contrast at all distances except those greater than 90 meters and at 40 and 20 meters to the intersection, respectively. At the questionnaire rating location, the pedestrian was always rendered in positive contrast in the Approach and Both lighting configurations, and in negative contrast in the Box lighting configuration (Figure 16).
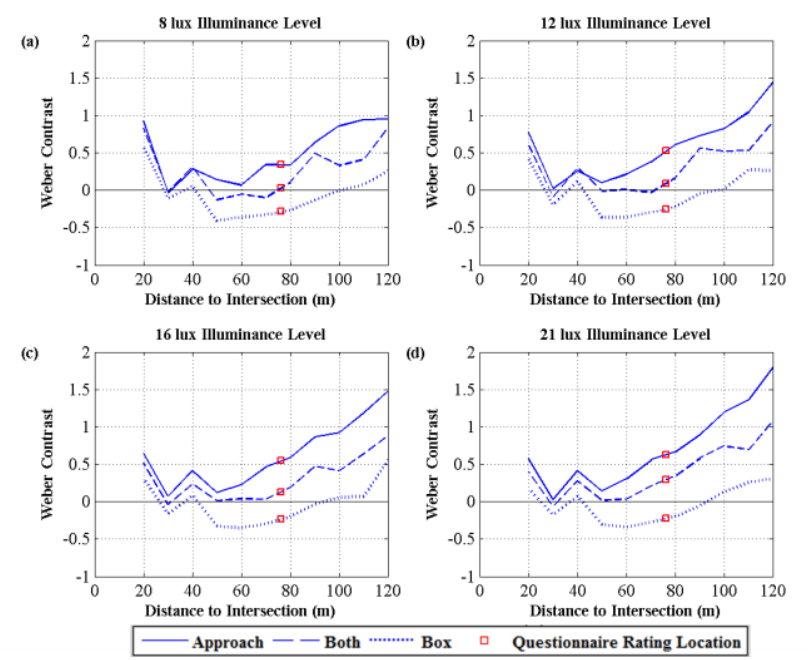

Figure 16. Pedestrian contrast as a function of the vehicle distance to the intersection. The red square represents the contrast of the pedestrian when viewed from the vehicle at the questionnaire rating location $(76.2 \mathrm{~m}$ from the intersection).

\section{Discussion}

Four major findings are evident from this work. First, target and pedestrian contrast and luminance were substantially affected by the intersection lighting configuration, illuminance level, location at the intersection, and the distance of the vehicle from the intersection. Objects also underwent changes in contrast polarity (positive to negative or vice-versa) as the distance between the vehicle and object changed. Second, targets in negative contrast were detected from longer distances than those in positive contrast. Third, within each contrast polarity, visual performance depended on the magnitude of contrast, with higher contrast associated with longer detection distances. Fourth, the relationship between pedestrian contrast and perceived visibility was complex, and a definitive conclusion could not be drawn regarding the effect of pedestrian contrast polarity on perceived pedestrian visibility.

Targets used in this study, placed at several locations within an intersection, underwent important changes in luminance and contrast as a vehicle approached the intersection. In general, target luminances increased as the vehicle got closer, most likely because targets entered the effective range of the vehicle headlamps. Increases in target luminance were not uniform, however, with target locations on the right hand side of the road (near right (Figure 5), near middle (Figure 6) and far right (Figure 8)) experiencing a larger increase in luminance compared to those on the left hand side (near left (Figure 7) and far left (Figure 9)). This difference could be attributed to the headlamp beam patterns, which are tailored to reduce luminous intensity in the left lane to reduce glare for oncoming drivers [20, 22]. Contrasts of targets at the locations used here also decreased until about 80 meters to the intersection. From closer than 80 meters, as the vehicle approached the intersection target contrasts increased as they entered the effective range of the vehicle headlamps and the luminance on the face of the target increased. This trend of increasing contrasts was clearly evident for the near right and near middle target locations (see Figure 10 and Figure 11), again because of the headlamp beam pattern.

Intersection lighting configurations also greatly influenced the magnitude and the polarity of contrast in which a target was rendered, 
as did the location of the target. In the Box lighting configuration, near right and near left targets were initially rendered in negative contrast. These changed into positive contrasts as the targets became within the effective range of the vehicle headlamps, which increased the luminance on the face of the target resulting in higher contrast magnitudes (see Figure 10 and Figure 11). During these polarity transitions, objects became invisible because the luminance of the target was equal to the luminance of the background resulting the contrast being zero (see Figure 17). In the Approach and Both lighting configurations, near right and near middle targets were initially rendered in positive contrasts, but also underwent an increase in the magnitude as the vehicle approached the intersection and the targets were in the effective range of the headlamps. The same phenomenon of increases in the contrast magnitude was observed for the far right and far left targets, which were rendered in positive contrast in all three lighting configurations (see Figure 13 and Figure 14).

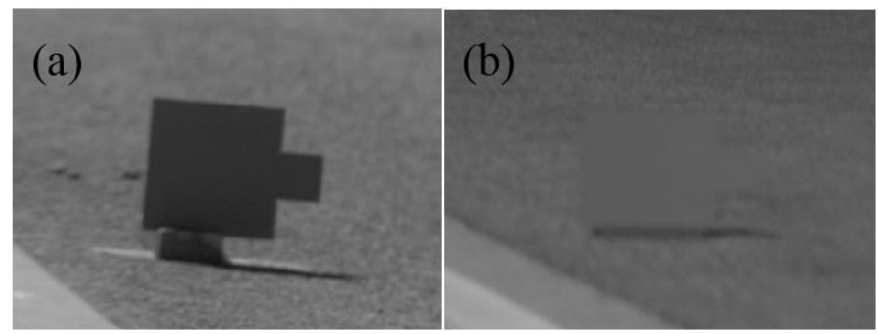

Figure 17. Target in negative contrast (a). Target approaching contrast neutrality where the luminance on the target is equal to the luminance of the background, thus making the target invisible.

Targets rendered in negative contrast had longer detection distances than those rendered in positive contrast as evidenced by the significantly longer mean detection distances of near right and near middle targets in the Box lighting configuration compared to the Approach lighting configuration, where the near right and near middle targets were rendered in positive contrast. Thus, negative contrast aided in increasing visual performance, which is in agreement with existing evidence that objects in negative contrast are detected sooner and from farther than objects in positive contrast [10, 12]. Furthermore, the longest mean detection distances for targets rendered in positive contrast were shorter than mean detection distances of targets rendered in negative contrast (see Figure 18). For a target rendered in positive contrast to be detected at distances comparable to that of a target in negative contrast, the magnitude of the positive contrast required is quite high (Figure 14b-d). For targets rendered in the same contrast polarity, visual performance depended on the magnitude of the contrast. For example, the far left and far right target locations were rendered in positive contrast in the Box lighting configuration, but the former was rendered in higher magnitude of contrast ( 8 lux -5.6 vs. $1.3,12$ lux -7.3 vs. $4.3,16$ lux -9.1 vs. 5.1 and 21 lux -10.3 vs. 6.4 ). Along with a higher contrast for the far left target location, the far left target had significantly longer mean detection distances ( 8 lux -89.9 vs. $60 \mathrm{~m}, 12$ lux 123.7 vs. $91.1 \mathrm{~m}, 16$ lux -126.2 vs. 90.5 and 21 lux -119.5 vs. 97.2 $\mathrm{m})$.

Page 8 of 10

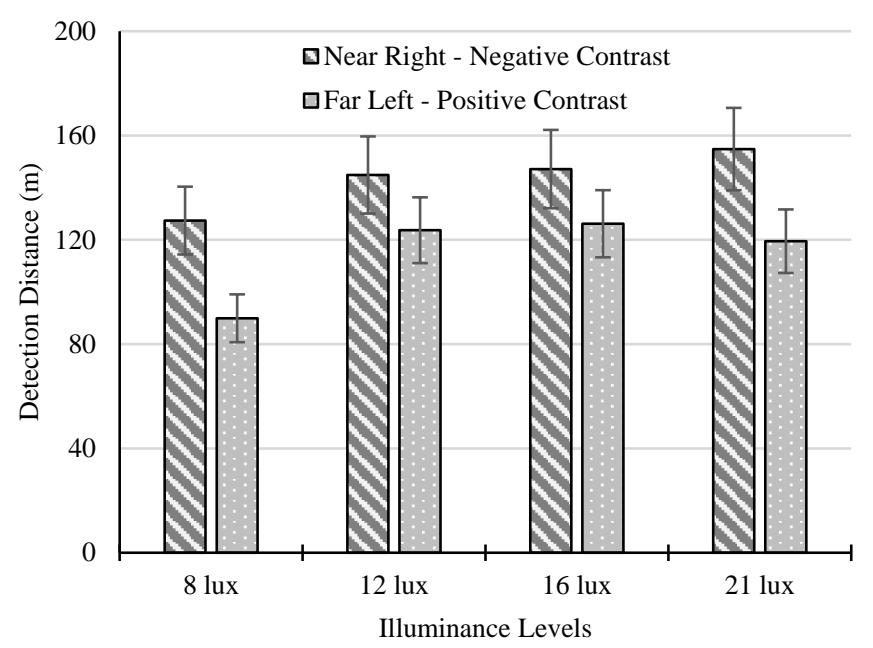

Figure 18. Mean detection distances of near right (negative contrast) and far left (positive contrast) target locations in the Box lighting configuration. Error bars reflect standard errors.

From the luminance measurements it was evident that the impact of headlamps begins from $\sim 80$ meters and increases rapidly as the vehicle gets closer to the intersection. Target locations in a lighting configuration with greater than 80 meter detection distances thus had minimal support from headlamp illumination during the target detection task. Such targets were apparently rendered in sufficient contrast (positive or negative) to be detected without headlamp support. Such an assessment was applied to each lighting configuration to determine if individual target locations were rendered in sufficient contrast. There were 20 total conditions (five target locations in each of four illuminance levels) in each lighting configuration.

In the Approach lighting configuration, the mean detection distance for every condition was less than 80 meters, indicating that the targets were detected only after they were in the range of headlamps. Approach lighting thus did not render the targets in sufficient positive contrast to facilitate detection. In the Box lighting configuration, a majority of conditions (18 out of 20) had mean detection distances greater than 80 meters, indicating that they were rendered at magnitudes of positive or negative contrasts that facilitated detection beyond the range of headlamps. In the Both lighting configuration, more than half (11 out of 20) of the target locations had mean detection distances less than 80 meters, indicating that headlamps were required to increase contrast and facilitate detection. Thus, in the Box lighting configuration, while negative contrast helped in the detection of near right and near middle target locations, targets rendered in positive contrast had higher magnitudes of contrast compared to the contrasts of similar targets in remaining lighting configurations to facilitate better visual performance. These results are also reinforced by the perceived visibility of targets, where the Box lighting configuration had the highest mean ratings. Overall, intersection lighting configuration with an illuminated box rendered targets at sufficient negative and positive contrast to facilitate better visual performance and higher perceptions of target visibility.

As was the case for targets, pedestrians also underwent major changes in luminance and contrast as the vehicle approached the intersection. As the vehicle approached the intersection (less than 60 meters), the increase in luminance was more rapid that for targets, as 
the pedestrian was in the effective range of the headlamps, which resulted in a higher rate of increase in the pedestrian luminance. Pedestrian contrast decreased as the vehicles neared the intersection, until the pedestrian was in the range of headlamps, after which the contrasts increased. The increase in the contrast is likely a result of the increase in luminance on the pedestrian from headlamp illumination.

Perceived pedestrian visibility was highest in the Approach lighting configuration, although there were no statistical differences in the perceived visibility ratings of pedestrians between the three lighting configurations. Differences in the perceptions of pedestrian and target visibility depended on the lighting configuration (and by extension object contrast, since the lighting configuration dictates the contrast in which an object is rendered) and object size. Within the Approach configuration, perceived pedestrian visibility was the highest and perceived target visibility was the lowest across all perceptual ratings. This result could be attributed to the object size, since the simulated pedestrian used was substantially larger than the targets, and there is a direct relationship between object size and perceived visibility [13]. However, our photometric measurements also indicate that contrast polarity has a differential effect on the perceived visibility of objects of different sizes. Negative contrast appears preferable for smaller objects, whereas positive contrast is preferable for larger objects (such as pedestrians).

Green [23] also noted that pedestrians might require positive contrast, to help in discerning features such as the face, hands, etc., versus negative contrast where the pedestrian appears as a silhouette and feature determination could be difficult. However, the current photometric assessments of a pedestrian in different intersection lighting configurations shows that determining pedestrian contrast polarity is complex. Pedestrians were often rendered in multiple contrast polarities (i.e., pedestrian's feet rendered in negative contrast and torso rendered in positive contrast, irrespective of lighting configuration; Figure 19). This difference makes it extremely difficult to determine if perceived pedestrian visibility was dependent on one or the other body region. On the contrary, targets, because of their smaller size, were not rendered in multiple contrast polarities (see Figure 19), and for these it was easier to relate target contrast with visual performance and perceived visibility. Further research is recommended to better understand the relationship between pedestrian contrast and perceived visibility.

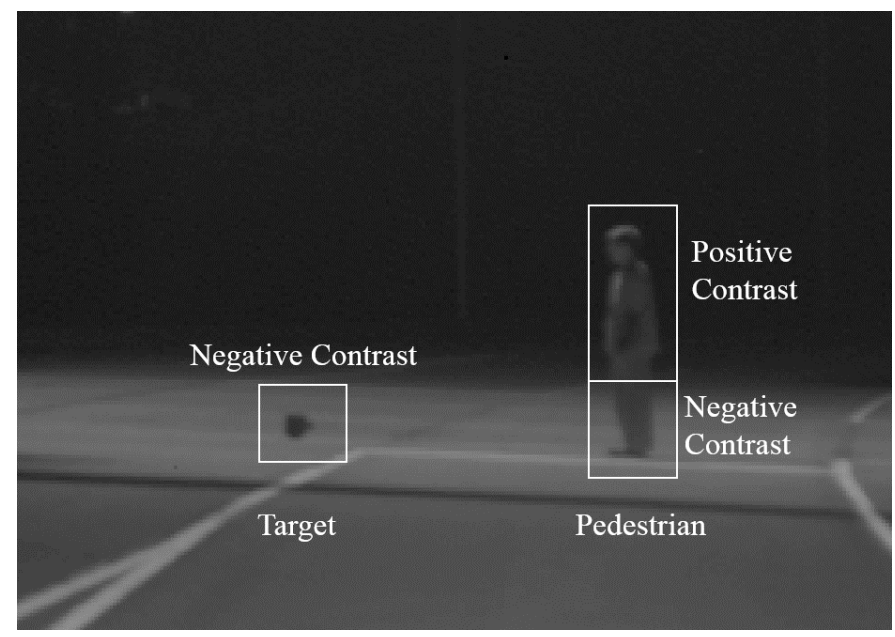

Figure 19. Contrast variance across objects. The pedestrian is rendered in multiple contrasts whereas there is no such variance for the smaller target
Changes in the contrast polarity of targets and pedestrian also has important implications for pedestrian detection systems that use computer/machine vision techniques. Since computer/machine vision technologies rely on visible light for pedestrian detection, they are also significantly affected by the same limitations as human eyes. In situations similar to those used in the current study, when the vehicle is at a certain distance from the object (pedestrian or target) under the interactive effects of vehicle headlamps and road lighting, the contrast of the object could become zero and objects cannot be visible, thus making them very difficult to detect. For optimal performance at night, a pedestrian detection system should be able to detect a pedestrian and track them on the vehicle's approach to avoid collisions. If, under the influence of headlamps and road lighting, the pedestrian is rendered invisible, it might become extremely difficult for a pedestrian detection system to track pedestrians and other hazards, thus adversely affecting system performance. For pedestrian detection systems to perform optimally at night, they should thus consider using alternative approaches, such as measuring color contrast, information from secondary sensors (e.g., radar), or infrared, in addition to using luminance contrast.

This study has two important limitations that should be noted. First, only grey colored targets and pedestrians were used for measuring luminance and contrast. In actual nighttime road scenarios, however, important objects can have different colors, pedestrians wear different colored clothing, and both targets and pedestrians may not be stationary. Second, we did not account for the presence of other vehicles/headlamps or the presence of continuous lighting of the roads leading to an intersection. These factors might introduce additional sources of light, which might affect the luminance and contrast of targets and pedestrians. While the current work helps to isolate important factors that affect object contrast, future work is needed to better understand the relationships between object contrast, visual performance, and perceived visibility under more diverse conditions.

\section{Conclusions}

In summary, road lighting and vehicle headlamps have important influences on the luminance and contrast of both targets and pedestrians. Target and pedestrian luminance increased as the vehicle approached the intersection. Object contrast varied not only with distance to the intersection, but also with the location of the target within an intersection. Target and pedestrian contrasts were also affected the by vehicle headlamps. Targets rendered in negative contrast had longer mean detection distances than those in positive contrast. The lighting configuration that illuminated the intersection box rendered almost all the target locations in sufficient positive and negative contrast to result in longer detection distances and higher perceived visibility. The relationship between pedestrian contrast and perceived pedestrian visibility was more complex, as pedestrians were rendered in multiple contrast polarities. Targets and pedestrians also underwent changes in contrast polarity (positive to negative or vice-versa) as the vehicle got closer to their locations. During these polarity transitions, target and pedestrian contrasst could become zero, rendering them invisible at certain distances. These findings have important implications for the lighting design of isolated/rural intersections and of pedestrian detection systems that rely on computer/machine vision. Illuminating the intersection box renders the targets located at the entrances, exits, and middle of crosswalks in sufficient contrast (both negative and positive) to ensure higher driver visual performance and perceived visibility compared to lighting designs that illuminate just the approach or both the approach and box.

Page 9 of 10 


\section{References}

1. Bullough, J. and M. Rea, Visual Performance Under Mesopic Conditions: Consequences for Roadway Lighting. Transportation Research Record: Journal of the Transportation Research Board, 2004. 1862(-1): p. 89-94.

2. Plainis, S., I.J. Murray, and W.N. Charman, The Role of Retinal Adaptation in Night Driving. Optometry \& Vision Science, 2005. 82(8): p. 682-688.

3. Várady, G. and P. Bodrogi, Mesopic spectral sensitivity functions based on visibility and recognition contrast thresholds. Ophthalmic and Physiological Optics, 2006. 26(3): p. 246-253.

4. Eloholma, M., et al., Visual performance in night-time driving conditions. Ophthalmic and Physiological Optics, 2006. 26(3): p. 254-263.

5. Lewis, A.L., Visual Performance as a Function of Spectral Power stribution of Light Sources at Luminances Used for Genreal Outdoor Lighting. Journal of the Illuminating Engineering Society, 1999. 28(1): p. 27-32.

6. Gibbons, R., B., et al., Evaluation of the Impact of Spectral Power Distribution on Driver Performance. 2015, Virginia Tech Transportation Institute.

7. Lingard, R. and M. Rea, Off-axis detection at mesopic light levels in a driving context. Journal of the Illuminating Engineering Society, 2002. 31(1): p. 33-39.

8. Alferdinck, J.W., Target detection and driving behaviour measurements in a driving simulator at mesopic light levels. Ophthalmic and physiological optics, 2006. 26(3): p. 264-280.

9. Akashi, Y. and M. Rea, Peripheral detection while driving under a mesopic light level. Journal of the Illuminating Engineering Society, 2002. 31(1): p. 85-94.

10. Aulhorn, E., Über die Beziehung zwischen Lichtsinn und Sehschärfe. Albrecht von Graefes Archiv für Ophthalmologie, 1964. 167(1): p. 4-74.

11. Adrian, W., Visibility of targets: Model for calculation. Lighting Research and Technology, 1989. 21(4): p. 181-188.

12. Hills, B.L., Visibility under night driving conditions: Part 2. Field measurements using disc obstacles and a pedestrian dummy. Lighting Research and Technology, 1975. 7(4): p. 251-258.

13. Janoff, M., Subjective ratings of visibility and alternative measures of roadway lighting. Journal of the Illuminating Engineering Society, 1989. 18(1): p. 16-28.

14. Ekrias, A., M. Eloholma, and L. Halonen, Effects of Vehicle Headlights on Target Contrast in Road Lighting Environments. Journal of Light \& Visual Environment, 2008. 32(3): p. 302-314.

15. Bhagavathula, R., R.B. Gibbons, and M.A. Nussbaum, Effects of Intersection Lighting Design on Nighttime Visual Performance of Drivers. LEUKOS, 2018. 14(1): p. 25-43.

16. Bhagavathula, R., R. Gibbons, and M.A. Nussbaum, Effect of Intersection Lighting Design on Drivers' Perceived Visibility and Glare. Transportation Research Record, 2019. 2673(2): p. 799810.

17. Pretto, P. and A. Chatziastros. Changes in optic flow and scene contrast affect the driving speed. in Driving Simulation Conference Europe. 2006.
18. Gibbons, R.B., et al., Informational report on lighting design for midblock crosswalks. 2008.

19. IES, RP-8-14 American National Standard Practice for Roadway Lighting. 2014.

20. Wördenweber, B., et al., Automotive Lighting and Human Vision. 2007: Springer.

21. Terry, T.N. and R.B. Gibbons. Road-Surface Contrast and Adaptation of Visibility of Low-Contrast Targets. in Transportation Research Board 94th Annual Meeting. 2015.

22. Boyce, P.R., Lighting for driving roads, vehicles, signs, and signals. 2009, Boca Raton: CRC Press.

23. Green, M., Forensic vision; with applications to highway safety, $3 d$ ed. (CD-ROM included). Scitech Book News. Vol. 32. 2008, Portland: Ringgold Inc.

\section{Contact Information}

Rajaram Bhagavathula, Ph.D., Virginia Tech Transportation Institute 3500 Transportation Research Plaza, Blacksburg, VA 24061 Work phone: (540) 231-5209

Email: rajaramb@vt.edu

\section{Acknowledgments}

The authors acknowledge the support of the stakeholders of the National Surface Transportation Safety Center for Excellence (NSTSCE): Tom Dingus from the Virginia Tech Transportation Institute, John Capp from General Motors Corporation, Chris Hayes from Travelers Insurance, Martin Walker from the Federal Motor Carrier Safety Administration, and Cathy McGhee from the Virginia Department of Transportation and the Virginia Center for Transportation Innovation and Research. The NSTSCE stakeholders have jointly funded this research for the purpose of developing and disseminating advanced transportation safety techniques and innovations.

\section{Definitions/Abbreviations}

LED

Light-emitting diode

IESNA

Illumination Engineering Society of North America

Page 10 of 10 